\title{
The Nadarajah Haghighi Topp Leone-G Family of Distributions with Mathematical Properties and Applications
}

\author{
Hesham Reyad \\ Department of Information Systems and Production Management \\ Qassim University, Kingdom of Saudi Arabia \\ Email: hesham_reyad@yahoo.com \\ Mahmoud Ali Selim \\ Community College, King Khalid University, Kingdom of Saudi Arabia \\ Faculty of Commerce, Al-Azhar University, Egypt \\ Email: selim.one@gmail.com
}

Soha Othman

Department of Applied Statistics, Cairo University, Egypt

Email: soha_othman@yahoo.com

\begin{abstract}
Based on the Nadarajah Haghighi distribution and the Topp Leone-G family in view of the T-X family, we introduce a new generator of continuous distributions with three extra parameters called the Nadarajah Haghighi Topp Leone-G family. Three sub-models of the new class are discussed. Main mathematical properties of the new family are investigated such as; quantile function, raw and incomplete moments, Bonferroni and Lorenz curves, moment and probability generating functions, stress strength model, Shanon and Rényi entropies, order statistics and probability weighted moments. The model parameters of the new family is estimated by using the method of maximum likelihood and the observed information matrix is also obtained. We introduce two real applications to show the importance of the new family.
\end{abstract}

Keywords: Entropies, Nadarajah Haghighi distribution, Maximum Likelihood, Probability Weighted Moments, Stress Strength Model, T-X Family.

\section{Introduction}

In the last decade, the famous distributions such as Lomax, Pareto, Gumbel, Lindley,...etc are extensively used for fitting data in various sciences such as medicine, engineering, agriculture and economics. However, in many applied areas such as environmental sciences, reliability studies and finance, there exist a great need to generalize these distributions to gain more acuracyand flexibility. Based on this reason, the statistican propose new methods for generating new generators of distributions to gain more desirable properties in the resulting distributions. Some of the recent families are : The transmuted Gompertz-G by Reyad et al. (2018a), Marshall-Olkin generalized G Poisson by Korkmaz et al. (2018a), generalized odd Weibull-G by Korkmaz et al. (2018b), generalized transmuted Poisson-G by Yousof et al.(2018), exponentiated odd log-LogisticG by Alizadeh et al. (2018), Topp Leone odd Lindley-G by Reyad et al. (2018b), extended odd Frechet by Yousof et al.(2019), transmuted generalized odd generalized exponentialG by Reyad et al. (2019a), odd Lomax-G by Cordeiro et al. (2019), Marshall-Olkin alpha 
power-G by Mazen er al. (2019), exponentiated generalized Topp Leone-G by Reyad et al. (2019b) odd inverse Pareto-G by Aldahlan et al. (2019); among others.

Let $g(x ; \phi)$ and $G(x ; \phi)$ denote the probability density function (pdf) and cumulative distribution function (cdf) of a baseline model with parameter vector $\phi$. Al-Shomrani et al. (2016) proposed the Topp Leone-G (TL-G) family of distributions with cdf by

$$
H(x ; \phi)=\left\{1-\bar{G}(x ; \phi)^{2}\right\}^{\theta}, \quad \theta>0, \quad x \in R,
$$

where, $\bar{G}(x ; \phi)=1-G(x ; \phi)$. Moreover, the Nadarajah Haghighi distribution has pdf given by

$$
f(t)=\alpha \lambda(1+\lambda t)^{\alpha-1} e^{1-(1+\lambda t)^{\alpha}}, x>0, \alpha, \lambda>0
$$

Based on the idea of T-X family pioneerd by Alzaatreh et al. (2013), TL-G class and the Nadarajah Haghighi distribution, we introduce a new class of continuous distributions called the Nadarajah Haghighi Topp Leone-G (NHTL-G for short) family with cdf given by

$$
\begin{aligned}
F(x ; \alpha, \lambda, \theta, \phi) & =\int_{0}^{H(x ; \phi)} f(t) d t \\
& =\int_{0}^{\left\{1-\bar{G}(x ; \phi)^{2}\right\}^{\theta}} \alpha \lambda(1+\lambda t)^{\alpha-1} e^{1-(1+\lambda t)^{\alpha}} d t \\
& =1-e^{1-\left\{1+\lambda\left(1-\bar{G}(x ; \phi)^{2}\right)^{\theta}\right\}^{\alpha}}, x \in R,
\end{aligned}
$$

The pdf of the NHTL-G family is given by

$$
\begin{aligned}
f(x ; \alpha, \lambda, \theta, \phi) & =2 \alpha \lambda \theta g(x ; \phi) \bar{G}(x ; \phi)\left(1-\bar{G}(x ; \phi)^{2}\right)^{\theta-1} \\
& \times\left\{1+\lambda\left(1-\bar{G}(x ; \phi)^{2}\right)^{\theta}\right\}^{\alpha-1} e^{1-\left\{1+\lambda\left(1-\bar{G}(x ; \phi)^{2}\right)^{\theta}\right\}^{\alpha}}, x \in R,
\end{aligned}
$$

We will denote a random variable $X$ with pdf (4) by X NHTL-G $(\alpha, \lambda, \theta, \varnothing)$. The hazard function $\tau(x)$ for the NHTL-G family is given by

$$
\begin{gathered}
\tau(x ; \alpha, \lambda, \theta, \phi)=2 \alpha \lambda \theta g(x ; \phi) \bar{G}(x ; \phi)\left[1-\bar{G}(x ; \phi)^{2}\right]^{\theta-1}\left\{1+\lambda\left[1-\bar{G}(x ; \phi)^{2}\right]^{\theta}\right\}^{\alpha-1} \\
\times\left\{1+\lambda\left[1-\bar{G}(x ; \phi)^{2}\right]^{\theta}\right\}^{\alpha-1}, x \in R
\end{gathered}
$$

The quantile function of the NHTL-G family, say $Q(u)=F^{-1}(u)$, for $u \in(0,1), \alpha \neq 0, \lambda \neq 0$ and $\theta \neq 0$ is the solution of the non-linear equation 


$$
Q(u)=G^{-1}\left(1-\left\{1-\left[\frac{1}{\lambda}\left\{[1-\log (1-u)]^{1 / \alpha}-1\right\}\right]^{1 / \theta}\right\}^{1 / 2}\right)
$$

This paper is organized as follows: An important expansions of the pdf and cdf corresponding to the new family are derived in Section 2. In Section 3, three special submodels of the NHTL-G family are discussed. Main mathematical properties of the NHTL$\mathrm{G}$ family are studies in Section 4. The model parameters of the new generator is estimated by using the method of maximum likelihood and the observed information matrix is also obtained in Section 5. In Section 6, two real applications of the NHTL-G are introduced. Concluding remarks are given in Section 7.

\section{Linear Representation}

In this section, we obtain the pdf and cdf of the new family as mixture repsentations of the exponentiated-G distribution. The pdf (4) can be expressed as

$$
\begin{aligned}
& f(x)=2 \alpha \lambda \theta e \sum_{w=0}^{\infty} \frac{(-1)^{w}}{w !} g(x ; \phi) \bar{G}(x ; \phi)\left[1-\bar{G}(x ; \phi)^{2}\right]^{\theta-1}\left\{1+\lambda\left[1-\bar{G}(x ; \phi)^{2}\right]^{\theta}\right\}^{\alpha(w+1)-1} \\
&=2 \alpha \lambda \theta e \sum_{w=0}^{\infty} \sum_{j=0}^{\infty} \frac{(-1)^{w} \lambda^{j}}{w !}\left(\begin{array}{c}
\alpha(w+1)-1 \\
j
\end{array}\right) g(x ; \phi) \bar{G}(x ; \phi)\left[1-\bar{G}(x ; \phi)^{2}\right]^{\theta(j+1)-1} \\
&=2 \alpha \lambda \theta e \sum_{w=0}^{\infty} \sum_{j=0}^{\infty} \sum_{i=0}^{\infty} \frac{(-1)^{w+i} \lambda^{j}}{w !}\left(\begin{array}{c}
\alpha(w+1)-1 \\
j
\end{array}\right) \\
& \times\left(\begin{array}{c}
\theta(j+1)-1 \\
i
\end{array}\right) g(x ; \phi)[1-G(x ; \phi)]^{2 i+1} \\
&= 2 \alpha \theta e \sum_{w=0}^{\infty} \sum_{j=0}^{\infty} \sum_{i=0}^{\infty} \sum_{\ell=0}^{2 i+1} \frac{(-1)^{w+i+\ell} \lambda^{j+1}}{w !}\left(\begin{array}{c}
\alpha(w+1)-1 \\
w
\end{array}\right) \\
& \times\left(\begin{array}{c}
\theta(j+1)-1 \\
i
\end{array}\right)\left(\begin{array}{c}
2 i+1 \\
\ell
\end{array}\right) g(x ; \phi) G(x ; \phi)^{\ell}
\end{aligned}
$$

Or equivalently

$$
f(x)=\sum_{\ell=0}^{2 i+1} \delta_{\ell} \pi_{\ell+1}(x)
$$


where, $\delta_{\ell}=2 \alpha \theta e \sum_{w=0}^{\infty} \sum_{j=0}^{\infty} \sum_{i=0}^{\infty} \frac{(-1)^{w+i+\ell} \lambda^{j+1}}{(\ell+1) w !}\left(\begin{array}{c}\alpha(w+1)-1 \\ j\end{array}\right)\left(\begin{array}{c}\theta(j+1)-1 \\ i\end{array}\right)\left(\begin{array}{c}2 i+1 \\ \ell\end{array}\right)$

and $\pi_{\ell+1}(x)=(\ell+1) g(x) G(x)^{\ell}$ is the exponentiated-G distribution with power parameter $\ell+1$.

Similarly, the cdf (3) can be written as

$$
F(x)=\sum_{\ell=0}^{2 i+1} \delta_{\ell} \Pi_{\ell+1}(x),
$$

where, $\Pi_{\ell+1}\left(x=G(x)^{\ell+1}\right.$.

\section{The Sub-Models of the New Family}

In this section, we introduce three special sub-models of the NHTL-G family.

\subsection{The NHTL-Lomax (NHTLLx) Model}

Suppose the cdf and pdf of the Lomax distribution are the following $G(x)=1-(1+b x)^{-a}, x \geq 0$, and $g(x)=a b\left((1+b x)^{-a}, x>0, a, b>0\right.$, respectively. Then, the pdf and cdf of NHTLLx distribution are given, respectively, by

$$
\begin{aligned}
f(x)= & 2 \alpha \lambda \theta a b(1+b x)^{-2 a-1}\left(1-(1+b x)^{-2 a}\right)^{\theta-1} \\
& \times\left\{1+\lambda\left(1-(1+b x)^{-2 a}\right)^{\theta}\right\}^{\alpha-1} e^{1-\left\{1+\lambda\left(1-(1+b x)^{-2 a}\right)^{\theta}\right\}^{\alpha}}, x>0 .
\end{aligned}
$$

and

$$
F(x)=1-e^{1-\left\{1+\lambda\left(1-(1+b x)^{-2 a}\right)^{\theta}\right\}^{\alpha}}, x \geq 0
$$

The plots of the density and hazard functions are given in Figure 1.
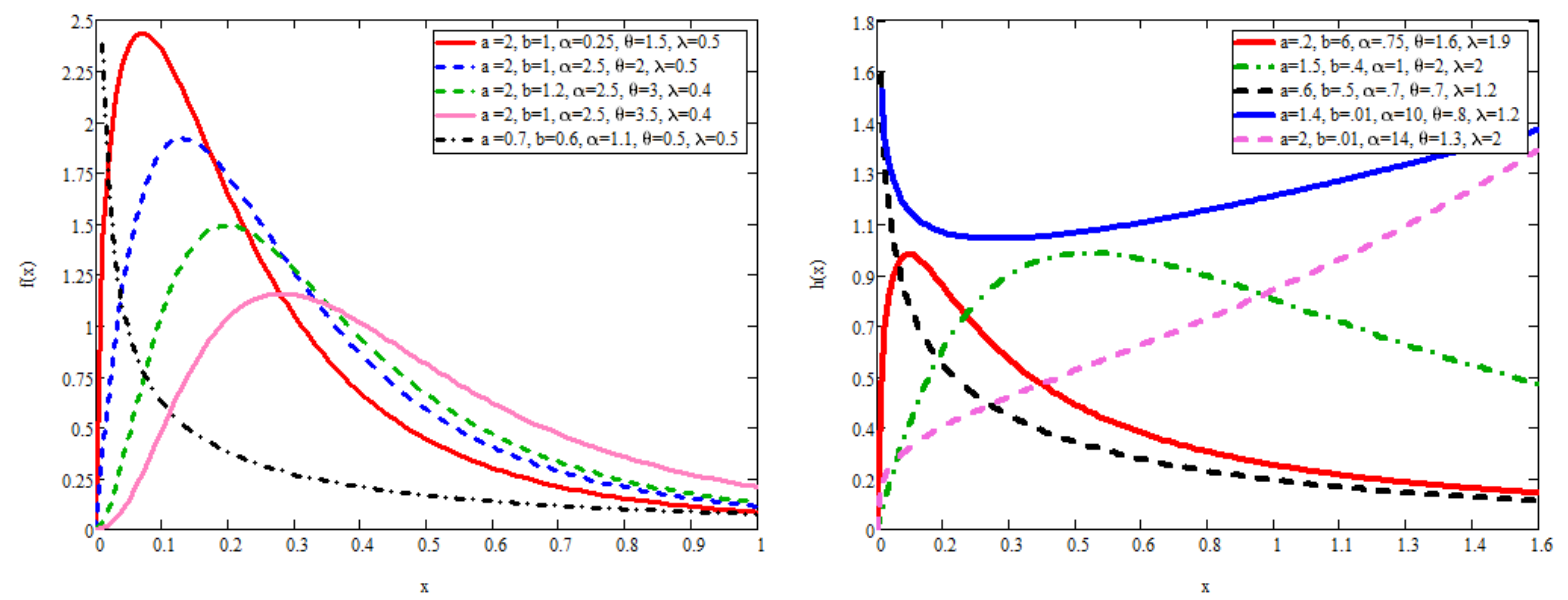

Figure 1: Plots of the NHTLLx pdf and hrf for selected values of parameters. 


\subsection{The NHTL-Kumaraswamy (NHTLKw) Model}

Consider the cdf and pdf of the Kumaraswamy distribution $G(x)=1-\left(1-x^{a}\right)^{b}, 0 \leq x \leq 1$, and $g(x)=a b x^{a-1}\left(1-x^{a}\right)^{b-1}, 0<x<1, a, b>0$, respectively. Then, the pdf and cdf of NHTLKw distribution are given, respectively, by

$$
\begin{aligned}
f(x)= & 2 \alpha \lambda \theta a b x^{a-1}\left(1-x^{a}\right)^{2 b-1}\left(1-\left(1-x^{a}\right)^{2 b}\right)^{\theta-1} \\
& \times\left\{1+\lambda\left(1-\left(1-x^{a}\right)^{2 b}\right)^{\theta}\right\}^{\alpha-1} e^{1-\left\{1+\lambda\left(1-\left(1-x^{a}\right)^{2 b}\right)^{\theta}\right\}^{\alpha}}, 0<x<1 .
\end{aligned}
$$

and

$$
F(x)=1-e^{1-\left\{1+\lambda\left(1-\left(1-x^{a}\right)^{2 b}\right)^{\theta}\right\}^{\alpha}}, 0 \leq x \leq 1
$$

The plots of the density and hazard functions are given in Figure 2.
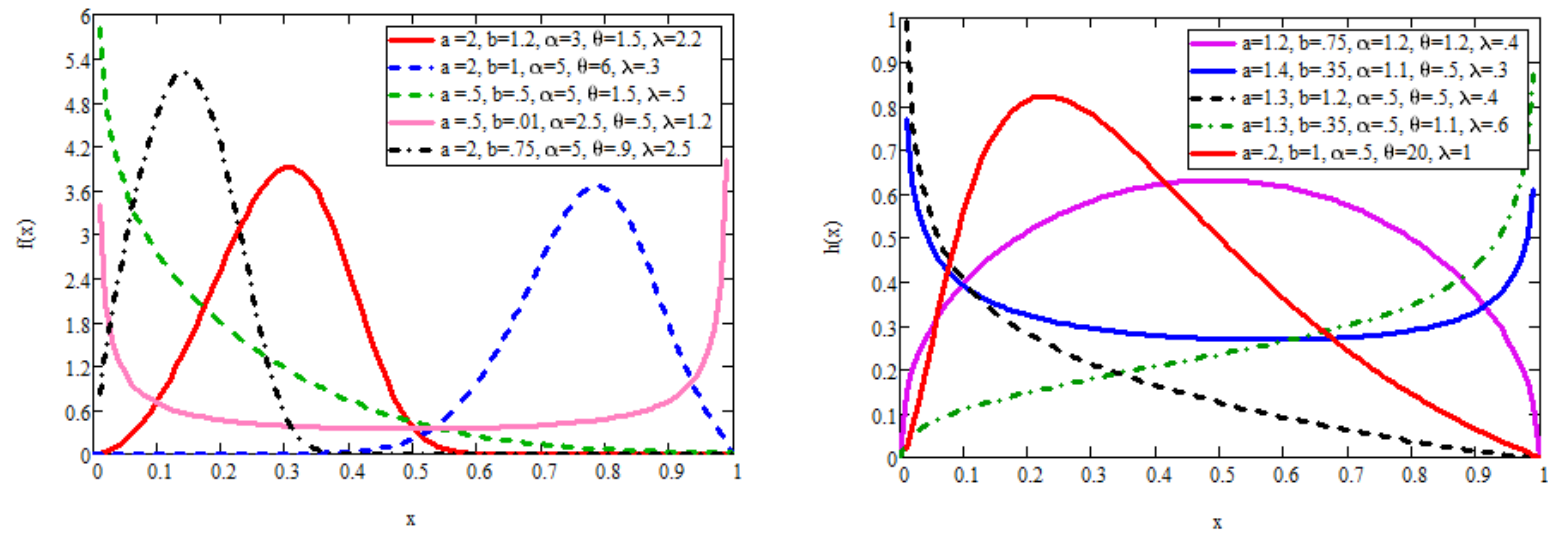

Figure 2: Plots of the NHTLKw pdf and hrf for selected values of parameters.

\subsection{The NHTL-Exponential (NHTLEx) Model}

Consider the cdf and pdf of the exponential distribution $G(x)=1-e^{-a x}, x \geq 0$, and $g(x)=a e^{-a x}, x>0, a>0$, respectively. Then, the pdf and cdf of NHTLEx distribution are given, respectively, by

$$
f(x)=2 \alpha \lambda \theta a e^{-2 a x}\left(1-e^{-2 a x}\right)^{\theta-1}\left\{1+\lambda\left(1-e^{-2 a x}\right)^{\theta}\right\}^{\alpha-1} e^{1-\left\{1+\lambda\left(1-e^{-2 a x}\right)^{\theta}\right\}^{\alpha}}, x>0 .
$$

and

$$
F(x)=1-e^{1-\left\{1+\lambda\left(1-e^{-2 a x}\right)^{\theta}\right\}^{\alpha}}, x \geq 0 .
$$

The plots of the density and hazard functions are given in Figure 2. 

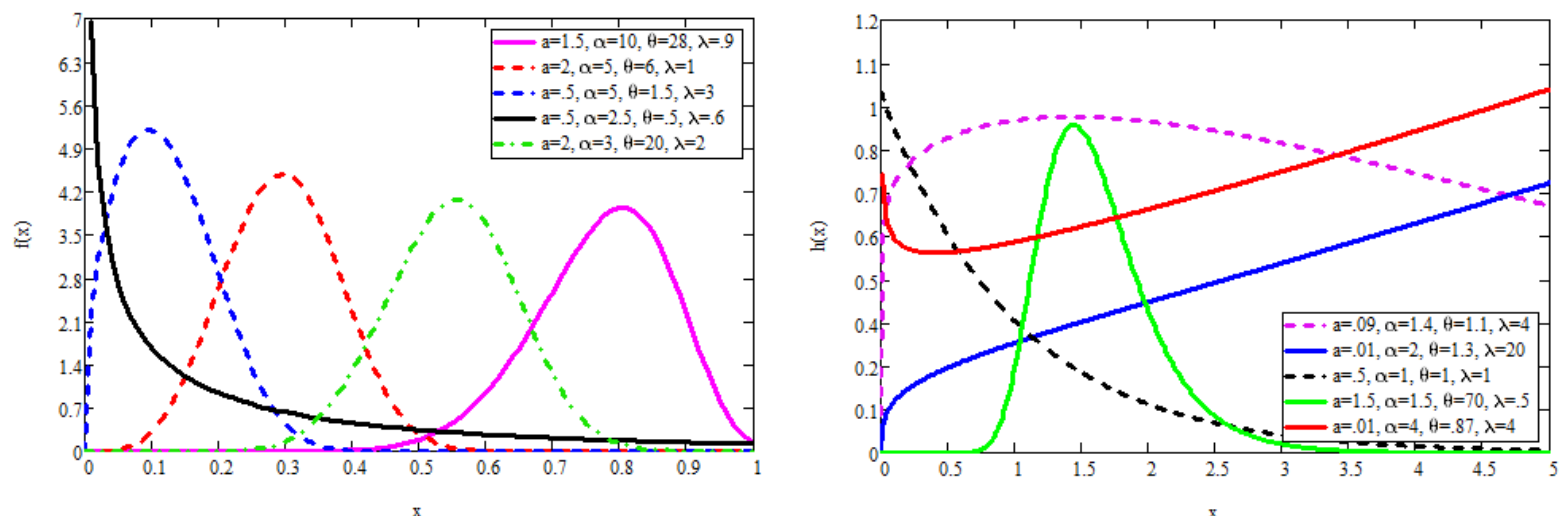

Figure 3: Plots of the NHTLEx pdf and hrf for selected values of parameters.

\section{Mathematical Properties}

This section studies some main properties of the NHTL-G family.

\subsection{Order Statistics}

Let $X_{1}, X_{2}, \ldots, X_{n}$ be a simple random sample from the NHTL-G family with cdf (3), pdf (4) and $X_{1: n}, X_{2: n}, \ldots, X_{n: n}$ be the corresponding order statistics. The pdf of $X_{k: n}$, the kth order statistic, is given by

$$
f_{X_{q: n}}(x)=\frac{1}{\beta(k, n-k+1)} \sum_{w=0}^{n-k}(-1)^{w}\left(\begin{array}{c}
n-k \\
w
\end{array}\right) f(x) F(x)^{k+w-1},
$$

where, $\beta(.,$.$) is the beta function. We can obtain from (3) and (4)$

$$
\begin{aligned}
& f(x) F(x)^{k+w-1}=2 \alpha \lambda \theta \sum_{j=0}^{k+w-1}(-1)^{j}\left(\begin{array}{c}
k+w-1 \\
j
\end{array}\right) g(x ; \phi) \bar{G}(x ; \phi)\left[1-\bar{G}(x ; \phi)^{2}\right]^{\theta-1} \\
& \times\left\{1+\lambda\left[1-\bar{G}(x ; \phi)^{2}\right]^{\theta}\right\}^{\alpha-1} e^{-(j+1)\left(1-\left\{1+\lambda\left[1-\bar{G}(x ; \phi)^{2}\right]^{\theta}\right\}^{\alpha}\right)} \\
& =2 \alpha \lambda \theta \sum_{j=0}^{k+w-1} \sum_{i=0}^{\infty} \frac{(-1)^{j+i}(j+1)^{i} e^{j+1}}{i !}\left(\begin{array}{c}
k+w-1 \\
j
\end{array}\right) \\
& \times g(x ; \phi) \bar{G}(x ; \phi)\left[1-\bar{G}(x ; \phi)^{2}\right]^{\theta-1}\left\{1+\lambda\left[1-\bar{G}(x ; \phi)^{2}\right]^{\theta}\right\}^{\alpha(i+1)-1} \\
& =2 \alpha \theta \sum_{j=0}^{k+w-1} \sum_{i, \ell=0}^{\infty} \frac{(-1)^{j+i}(j+1)^{i} e^{j+1} \lambda^{\ell+1}}{i !}\left(\begin{array}{c}
k+w-1 \\
j
\end{array}\right) \\
& \times\left(\begin{array}{c}
\alpha(i+1)-1 \\
\ell
\end{array}\right) g(x ; \phi) \bar{G}(x ; \phi)\left[1-\bar{G}(x ; \phi)^{2}\right]^{\theta(\ell+1)-1} \\
& =2 \alpha \theta \sum_{j=0}^{k+w-1} \sum_{i, \ell, d=0}^{\infty} \sum_{m=0}^{2 d+1} \frac{(-1)^{j+i+d+m}(j+1)^{i} e^{j+1} \lambda^{\ell+1}}{i !}\left(\begin{array}{c}
k+w-1 \\
j
\end{array}\right) \\
& \times\left(\begin{array}{c}
\alpha(i+1)-1 \\
\ell
\end{array}\right)\left(\begin{array}{c}
\theta(\ell+1)-1 \\
d
\end{array}\right)\left(\begin{array}{c}
2 d+1 \\
m
\end{array}\right) g(x ; \phi) \bar{G}(x ; \phi)^{m}
\end{aligned}
$$

Or equivalently, 


$$
f(x) F(x)^{k+w-1}=\sum_{m=0}^{2 d+1} \mu_{m} \pi_{m+1}(x)
$$

where, $\mu_{m}=2 \alpha \theta \sum_{j=0}^{k+w-1} \sum_{i, \ell, d=0}^{\infty} \frac{(-1)^{j+i+d+m}(j+1)^{i} e^{j+1} \lambda^{\ell+1}}{i !(m+1)}\left(\begin{array}{c}k+w-1 \\ j\end{array}\right)\left(\begin{array}{c}\alpha(i+1)-1 \\ \ell\end{array}\right)\left(\begin{array}{c}\theta(\ell+1)-1 \\ d\end{array}\right)\left(\begin{array}{c}2 d+1 \\ m\end{array}\right)$.

Substituting from (10) into (9), we arrive at

$$
f_{X_{k: n}}(x)=\sum_{m=0}^{2 d+1} \mu_{m}^{*} \pi_{m+1}(x)
$$

where, $\mu_{m}^{*}=\sum_{w=0}^{n-k} \frac{(-1)^{w}}{\beta(q, n-k+1)}\left(\begin{array}{c}n-k \\ w\end{array}\right) \mu_{m}$.

In addition, the $r$ th moment of the kth order statistic for NHTL-G family is given by

$$
E\left(x_{k: n}^{r}\right)=\sum_{m=0}^{2 d+1} \mu_{m}^{* *} \pi_{m+1}(x)
$$

where, $\mu_{m}^{* *}=(m+1) \mu_{m}^{*}$.

\subsection{Probability Weighted Moments}

The $(r+s)$ th PWM of $X$ with NHTL-G distribution denoted as $v_{r, s}$, is given by

$$
v_{r, s}=E\left(X^{r} F(x)^{s}\right)=\int_{-\infty}^{\infty} X^{r} F(x)^{s} f(x) d x .
$$

By using (3) and (4) and after some simplifications, we arrive at

$$
f(x) F(x)^{s}=\sum_{m=0}^{2 \ell+1} a_{m} \pi_{m+1}(x)
$$

where, $a_{m}=2 \alpha \theta \sum_{h=0}^{\infty} \sum_{j=0}^{\infty} \sum_{i=0}^{\infty} \sum_{\ell=0}^{\infty} \frac{(-1)^{h+j+\ell+m}(h+1)^{j} e^{h+1} \lambda^{i+1}}{i !(m+1)}\left(\begin{array}{l}s \\ h\end{array}\right)\left(\begin{array}{c}\alpha(j+1)-1 \\ i\end{array}\right)\left(\begin{array}{c}\theta(i+1)-1 \\ \ell\end{array}\right)\left(\begin{array}{c}2 \ell+1 \\ m\end{array}\right)$,

Substituting (14) into (13), we have

$$
v_{r, s}=\sum_{m=0}^{2 \ell+1} a_{m}^{*} \Psi_{r, m}
$$

where, $a_{m}^{*}=(m+1) a_{m}$ and $\Psi_{r, m}=\int_{-\infty}^{\infty} x^{r} g(x) G(x)^{m} d x$ is the probability weighted moment of the parent distribution.

\subsection{Moments}

The raw moment denoted as, $\mu_{r}^{\prime}$, of a random variable where, $X$ with NHTL-G distribution is given by

$$
\begin{aligned}
\mu_{r}^{\prime} & =E\left(X^{r}\right)=\int_{-\infty}^{\infty} x^{r} f(x) d x \\
& =\sum_{\ell=0}^{2 i+1} \delta_{\ell}^{*} \Psi_{r, \ell},
\end{aligned}
$$

where, $\delta_{\ell}^{*}=(\ell+1) \delta_{\ell}$. 
The nth central moment of the NHTL-G distribution, say $\mu_{n}$, can be calculated from

$$
\begin{aligned}
\mu_{n} & =\sum_{r=0}^{n}\left(\begin{array}{l}
n \\
r
\end{array}\right)\left(-\mu_{1}^{\prime}\right)^{n-r} E\left(x^{r}\right) \\
& =\sum_{\ell=0}^{2 i+1} \delta_{\ell}^{* *} \Psi_{r, \ell},
\end{aligned}
$$

where, $\delta_{\ell}^{* *}=\sum_{r=0}^{n}\left(\begin{array}{l}n \\ r\end{array}\right)\left(-\mu_{1}^{\prime}\right)^{n-r} \delta_{\ell}^{*}$.

The $r$ th incomplete moment of $X$, denoted by $\varphi_{s}(t)$, is

$$
\begin{aligned}
\varphi_{s}(t) & =\int_{-\infty}^{t} x^{s} f(x) d x \\
& =\sum_{\ell=0}^{2 i+1} \delta_{\ell}^{*} \Delta_{r, \ell},
\end{aligned}
$$

where, $\Delta_{r, \ell}=\int_{-\infty}^{t} x^{s} g(x) G(x)^{\ell} d x$.

The Lorenz and Bonferroni curves denoted as $L_{F}(x)$ and $B(F(x))$ can be calculated from (18) by using $L_{F}(x)=\varphi_{1}(t) / \mu_{1}^{\prime}$ and $B(F(x))=L_{F}(x) / F(x)$.

The moment and probability generating functions denoted as $M_{x}(t)$ and $M_{[x]}(t)$ of the NHTL-G distribution are given, respectively, by

$$
M_{x}(t)=E\left(e^{t x}\right)=\sum_{r=0}^{\infty} \sum_{\ell=0}^{2 i+1} \frac{t^{r}}{r !} \delta_{\ell}^{*} \Psi_{r, \ell},
$$

and

$$
M_{[x]}(t)=E\left(t^{x}\right)=\sum_{r=0}^{\infty} \sum_{\ell=0}^{2 i+1} \frac{(\ln t)^{r}}{r !} \delta_{\ell}^{*} \Psi_{r, \ell}
$$

\subsection{Entropies}

The Rényi entropy can be obtained from

$$
I_{R}(X)=(1-\beta)^{-1} \log \int_{-\infty}^{\infty} f(x)^{\beta} d x, \beta>0, \beta \neq 0 .
$$

Based on (4), we have

$$
f(x)^{\beta}=\sum_{\ell=0}^{\infty} k_{\ell} g(x)^{\beta} G(x)^{\ell},
$$

where, $k_{\ell}=(2 \alpha \theta e) \sum^{\beta} \sum_{w=0}^{\infty} \sum_{j=0}^{\infty} \sum_{i=0}^{\infty} \frac{(-1)^{w+i+\ell} \beta^{w} \lambda^{\beta+j}}{w !}\left(\begin{array}{c}\alpha(\beta+w)-\beta \\ j\end{array}\right)\left(\begin{array}{c}\theta(\beta+j)-\beta \\ i\end{array}\right)\left(\begin{array}{c}2 i+\beta \\ \ell\end{array}\right)$.

Inserting (22) into (21), we have

$$
I_{R}(X)=(1-\beta)^{-1} \log \left(\sum_{\ell=0}^{\infty} k_{\ell} \int_{-\infty}^{\infty} g(x)^{\beta} G(x)^{\ell} d x\right) .
$$


The Shannon entropy is defined as follows:

$$
\Upsilon_{x}=-E\{\log f(x)\} .
$$

From (4), we have

$$
\begin{aligned}
\log f(x)=1 & +\log (2 \alpha \lambda \theta)+\log (g(x ; \phi))-\log (\bar{G}(x ; \phi)) \\
& +(\theta-1) \log \left(1-\bar{G}(x ; \phi)^{2}\right)+(\alpha-1) \log \left\{1+\lambda\left(1-\bar{G}(x ; \phi)^{2}\right)^{\theta}\right\} \\
& -\left\{1+\lambda\left(1-\bar{G}(x ; \phi)^{2}\right)^{\theta}\right\}^{\alpha}
\end{aligned}
$$

Using $\log (1-z)=-\sum_{w=1}^{\infty} \frac{z^{w}}{w}$ and $\log (1+z)=-\sum_{w=1}^{\infty} \frac{(-1)^{w-1} z^{w}}{w},|z|<1$ in (25), we can obtain the following quantities:

$$
\begin{aligned}
& \log (\bar{G}(x ; \phi))=-\sum_{w=1}^{\infty} \frac{G(x ; \phi)^{w}}{w} \\
& \log \left(1-\bar{G}(x ; \phi)^{2}\right)=-\sum_{w=1}^{\infty} \sum_{j=0}^{2 w} \frac{(-1)^{j}}{w}\left(\begin{array}{c}
2 w \\
j
\end{array}\right) G(x ; \phi)^{j} \\
& \log \left\{1+\lambda\left(1-\bar{G}(x ; \phi)^{2}\right)^{\theta}\right\}=-\sum_{w=1}^{\infty} \sum_{i=0}^{\infty} \sum_{j=0}^{2 i} \frac{(-1)^{w+i+j-1} \lambda^{w}}{w}\left(\begin{array}{c}
\theta w \\
i
\end{array}\right)\left(\begin{array}{c}
2 i \\
j
\end{array}\right) G(x ; \phi)^{j} \\
& \left\{1+\lambda\left(1-\bar{G}(x ; \phi)^{2}\right)^{\theta}\right\}^{\alpha}=\sum_{m=0}^{\infty} \sum_{i=0}^{\infty} \sum_{j=0}^{2 i}(-1)^{i+j} \lambda^{m}\left(\begin{array}{c}
\alpha \\
m
\end{array}\right)\left(\begin{array}{c}
\theta m \\
i
\end{array}\right)\left(\begin{array}{c}
2 i \\
j
\end{array}\right) G(x ; \phi)^{j}
\end{aligned}
$$

Inserting the previous quantities into (25) and then into (24), we arrive at

$$
\begin{aligned}
\Upsilon_{x}= & -1-\log (2 \alpha \lambda \theta)-E(\log g(x ; \phi))+\sum_{w=1}^{\infty} \frac{E\left(G(x ; \phi)^{w}\right)}{w} \\
& +(\theta-1) \sum_{w=1}^{\infty} \sum_{j=0}^{2 w} \frac{(-1)^{j}}{w}\left(\begin{array}{c}
2 w \\
j
\end{array}\right) E\left(G(x ; \phi)^{j}\right) \\
& -(\alpha-1) \sum_{w=1}^{\infty} \sum_{i=0}^{\infty} \sum_{j=0}^{2 i} \frac{(-1)^{w+i+j-1} \lambda^{w}}{w}\left(\begin{array}{c}
\theta w \\
i
\end{array}\right)\left(\begin{array}{c}
2 i \\
j
\end{array}\right) E\left(G(x ; \phi)^{j}\right) \\
& +\sum_{m=0}^{\infty} \sum_{i=0}^{\infty} \sum_{j=0}^{2 i}(-1)^{i+j} \lambda^{m}\left(\begin{array}{c}
\alpha \\
m
\end{array}\right)\left(\begin{array}{c}
\theta m \\
i
\end{array}\right)\left(\begin{array}{c}
2 i \\
j
\end{array}\right) E\left(G(x ; \phi)^{j}\right) .
\end{aligned}
$$

\subsection{Stress Strength Model}

Let $X_{1}$ and $X_{2}$ be two independent random variables with NHTL- G $\left(\alpha_{1}, \lambda_{1}, \theta_{1}, \phi\right)$. and NHTL- $\mathrm{G}\left(\alpha_{2}, \lambda_{2}, \theta_{2}, \phi\right)$. distributions. Then, the stress strength model is given by

$$
R=\operatorname{Pr}\left(X_{2}<X_{1}\right)=\int_{0}^{\infty} f_{1}\left(\alpha_{1}, \lambda_{1}, \theta_{1}, \phi\right) F_{2}\left(\alpha_{2}, \lambda_{2}, \theta_{2}, \phi\right) d x .
$$

Equations (1) and (2) yield

$$
f_{1}\left(\alpha_{1}, \lambda_{1}, \theta_{1}, \phi\right) F_{2}\left(\alpha_{2}, \lambda_{2}, \theta_{2}, \phi\right)=2 e \alpha_{1} \lambda_{1} \theta_{1} g(x ; \phi) \bar{G}(x ; \phi)\left(1-\bar{G}(x ; \phi)^{2}\right)^{\theta_{1}-1}
$$




$$
\begin{aligned}
& \times\left\{1+\lambda_{1}\left(1-\bar{G}(x ; \phi)^{2}\right)^{\theta_{1}}\right\}^{\alpha_{1}-1} e^{-\left\{1+\lambda_{1}\left(1-\bar{G}(x ; \phi)^{2}\right)^{\theta_{1} \alpha_{1}}\right.} \\
& -2 e^{2} \alpha_{1} \lambda_{1} \theta_{1} g(x ; \phi) \bar{G}(x ; \phi)\left(1-\bar{G}(x ; \phi)^{2}\right)^{\theta_{1}-1} \\
& \times\left\{1+\lambda_{1}\left(1-\bar{G}(x ; \phi)^{2}\right)^{\theta_{1}}\right\}^{\alpha_{1}-1} \\
& \times e^{-\left\{1+\lambda_{1}\left(1-\bar{G}(x ; \phi)^{2}\right)^{\theta_{1}}\right\}^{\alpha_{1}}-\left\{1+\lambda_{2}\left(1-\bar{G}(x ; \phi)^{2}\right)^{\theta_{2}}\right\}^{\alpha_{2}}} \\
& =2 e \alpha_{1} \lambda_{1} \theta_{1} \sum_{w=0}^{\infty} \frac{(-1)^{w}}{w !} g(x ; \phi) \bar{G}(x ; \phi) \\
& \times\left(1-\bar{G}(x ; \phi)^{2}\right)^{\theta_{1}-1}\left\{1+\lambda_{1}\left(1-\bar{G}(x ; \phi)^{2}\right)^{\theta_{1}}\right\}^{\alpha_{1}(w+1)-1} \\
& -2 e^{2} \alpha_{1} \lambda_{1} \theta_{1} \sum_{w=0}^{\infty} \sum_{h=0}^{\infty} \frac{(-1)^{w+h}}{w ! h !} g(x ; \phi) \bar{G}(x ; \phi) \\
& \times\left(1-\bar{G}(x ; \phi)^{2}\right)^{\theta_{1}-1}\left\{1+\lambda_{1}\left(1-\bar{G}(x ; \phi)^{2}\right)^{\theta_{1}}\right\}^{\alpha_{1}(w+1)-1} \\
& \times\left\{1+\lambda_{2}\left(1-\bar{G}(x ; \phi)^{2}\right)^{\theta_{2}}\right\}^{\alpha_{2} h} \\
& =2 e \alpha_{1} \theta_{1} \sum_{w=0}^{\infty} \sum_{c=0}^{\infty} \frac{(-1)^{w} \lambda_{1}^{c+1}}{w !}\left(\begin{array}{c}
\alpha_{1}(w+1)-1 \\
c
\end{array}\right) g(x ; \phi) \\
& \times \bar{G}(x ; \phi)\left(1-\bar{G}(x ; \phi)^{2}\right)^{\theta_{1}(c+1)-1} \\
& -2 e^{2} \alpha_{1} \theta_{1} \sum_{w=0}^{\infty} \sum_{h=0}^{\infty} \sum_{c=0}^{\infty} \sum_{d=0}^{\infty} \frac{(-1)^{w+h} \lambda_{1}^{c+1} \lambda_{2}^{d}}{w ! h !} \\
& \times\left(\begin{array}{c}
\alpha_{1}(w+1)-1 \\
c
\end{array}\right)\left(\begin{array}{c}
\alpha_{2} h \\
d
\end{array}\right) g(x ; \phi) \\
& \times \bar{G}(x ; \phi)\left(1-\bar{G}(x ; \phi)^{2}\right)^{\theta_{1}(c+1)+\theta_{2} d-1} \\
& =2 e \alpha_{1} \theta_{1} \sum_{w=0}^{\infty} \sum_{c=0}^{\infty} \sum_{\ell=0}^{\infty} \frac{(-1)^{w+\ell} \lambda_{1}^{c+1}}{w !} \\
& \times\left(\begin{array}{c}
\alpha_{1}(w+1)-1 \\
c
\end{array}\right)\left(\begin{array}{c}
\theta_{1}(c+1)-1 \\
\ell
\end{array}\right) g(x ; \phi) \bar{G}(x ; \phi)^{2 \ell+1} \\
& -2 e^{2} \alpha_{1} \theta_{1} \sum_{w=0}^{\infty} \sum_{h=0}^{\infty} \sum_{c=0}^{\infty} \sum_{d=0}^{\infty} \sum_{\ell=0}^{\infty} \frac{(-1)^{w+h+\ell} \lambda_{1}^{c+1} \lambda_{2}^{d}}{w ! h !} \\
& \times\left(\begin{array}{c}
\alpha_{1}(w+1)-1 \\
c
\end{array}\right)\left(\begin{array}{c}
\alpha_{2} h \\
d
\end{array}\right)\left(\begin{array}{c}
\theta_{1}(c+1)+\theta_{2} d-1 \\
\ell
\end{array}\right) \\
& \times g(x ; \phi) \bar{G}(x ; \phi)^{2 \ell+1}
\end{aligned}
$$




$$
\begin{aligned}
=2 e \alpha_{1} \theta_{1} \sum_{w=0}^{\infty} \sum_{c=0}^{\infty} \sum_{\ell=0}^{\infty} & \sum_{m=0}^{2 \ell+1} \frac{(-1)^{w+\ell+m} \lambda_{1}^{c+1}}{w !}\left(\begin{array}{c}
\alpha_{1}(w+1)-1 \\
c
\end{array}\right) \\
& \times\left(\begin{array}{c}
\theta_{1}(c+1)-1 \\
\ell
\end{array}\right)\left(\begin{array}{c}
2 \ell+1 \\
m
\end{array}\right) g(x ; \phi) G(x ; \phi)^{m} \\
& -2 e^{2} \alpha_{1} \theta_{1} \sum_{w=0}^{\infty} \sum_{h=0}^{\infty} \sum_{c=0}^{\infty} \sum_{d=0}^{\infty} \sum_{\ell=0}^{\infty} \sum_{m=0}^{\infty} \frac{(-1)^{w+h+\ell+m} \lambda_{1}^{c+1} \lambda_{2}^{d}}{w ! h !} \\
& \times\left(\begin{array}{c}
\alpha_{1}(w+1)-1 \\
c
\end{array}\right)\left(\begin{array}{c}
\alpha_{2} h \\
d
\end{array}\right)\left(\begin{array}{c}
\theta_{1}(c+1)+\theta_{2} d-1 \\
\ell
\end{array}\right) \\
& \times\left(\begin{array}{c}
2 \ell+1 \\
m
\end{array}\right) g(x ; \phi) G(x ; \phi)^{m}
\end{aligned}
$$

Or

$$
f_{1}\left(\alpha_{1}, \lambda_{1}, \theta_{1}, \phi\right) F_{2}\left(\alpha_{2}, \lambda_{2}, \theta_{2}, \phi\right)=\sum_{m=0}^{2 \ell+1}\left(\varepsilon_{m}+\Omega_{m}\right) \pi_{m+1}(x),
$$

where, $\varepsilon_{m}=2 e \alpha_{1} \theta_{1} \sum_{w=0}^{\infty} \sum_{c=0}^{\infty} \sum_{\ell=0}^{\infty} \frac{(-1)^{w+\ell+m} \lambda_{1}^{c+1}}{(m+1) w !}\left(\begin{array}{c}\alpha_{1}(w+1)-1 \\ c\end{array}\right)\left(\begin{array}{c}\theta_{1}(c+1)-1 \\ \ell\end{array}\right)\left(\begin{array}{c}2 \ell+1 \\ m\end{array}\right)$,

and $\Omega_{m}=2 e^{2} \alpha_{1} \theta_{1} \sum_{w=0}^{\infty} \sum_{h=0}^{\infty} \sum_{c=0}^{\infty} \sum_{d=0}^{\infty} \sum_{\ell=0}^{\infty}\left(\begin{array}{c}\alpha_{1}(w+1)-1 \\ c\end{array}\right)\left(\begin{array}{c}\alpha_{2} h \\ d\end{array}\right)\left(\begin{array}{c}\theta_{1}(c+1)+\theta_{2} d-1 \\ \ell\end{array}\right)\left(\begin{array}{c}2 \ell+1 \\ m\end{array}\right)$.

Inserting (28) into (27), we arrive at

$$
R=\sum_{m=0}^{2 \ell+1}\left(\varepsilon_{m}+\Omega_{m}\right)
$$

\section{Estimation of Parameters}

This section deals with the maximum likelihood estimates (MLEs) of the model parameters of the NHTL-G distribution from complete samples. Let $x_{1}, x_{2}, \ldots, x_{n}$ be an observed values of a random sample from the NHTL-G family with parameter vector $\Phi=(\alpha, \lambda, \theta, \phi)^{T}$, then the log-likelihood function is given by

$$
\begin{aligned}
\ell= & n\{1+\log (2)+\log (\alpha)+\log (\lambda)+\log (\theta)\}+\sum_{i=1}^{n} \log \left(g\left(x_{i}, \phi\right)\right) \\
& +\sum_{i=1}^{n} \log \left(\bar{G}\left(x_{i}, \phi\right)\right)+(\theta-1) \sum_{i=1}^{n} \log \left(a_{i}\right)-\sum_{i=1}^{n}\left\{1+\lambda a_{i}^{\theta}\right\}^{\alpha} \\
& +(\alpha-1) \sum_{i=1}^{n} \log \left\{1+\lambda a_{i}^{\theta}\right\},
\end{aligned}
$$

where, $a_{i}=1-\bar{G}\left(x_{i}, \phi\right)^{2}$.

By differentiating (30) with respect to $\alpha, \lambda, \theta$ and $\phi$, we obtain the following nonlinear system of equations 


$$
\begin{aligned}
\frac{\partial \ell}{\partial \alpha}= & \frac{n}{\alpha}+\sum_{i=1}^{n} \log \left\{1+\lambda a_{i}^{\theta}\right\}-\sum_{i=1}^{n}\left\{\left(1+\lambda a_{i}^{\theta}\right) \log \left(1+\lambda a_{i}^{\theta}\right)\right\} \\
\frac{\partial \ell}{\partial \lambda}= & \frac{n}{\lambda}+(\alpha-1) \sum_{i=1}^{n}\left\{\frac{a_{i}^{\theta}}{1+\lambda a_{i}^{\theta}}\right\}-\alpha \sum_{i=1}^{n}\left\{a_{i}^{\theta}\left(1+\lambda a_{i}^{\theta}\right)^{\alpha-1}\right\} \\
\frac{\partial \ell}{\partial \theta} & =\frac{n}{\theta}+\sum_{i=1}^{n} \log \left(a_{i}\right)++\lambda(\alpha-1) \sum_{i=1}^{n}\left\{\frac{a_{i}^{\theta} \log \left(a_{i}\right)}{1+\lambda a_{i}^{\theta}}\right\} \\
& -\alpha \lambda \sum_{i=1}^{n}\left\{a_{i}^{\theta}\left(1+\lambda a_{i}^{\theta}\right)^{\alpha-1} \log \left(a_{i}\right)\right\}
\end{aligned}
$$

and

$$
\begin{aligned}
\frac{\partial \ell}{\partial \phi}= & \sum_{i=1}^{n}\left(\frac{g^{\prime}\left(x_{i}, \phi\right)}{g\left(x_{i}, \phi\right)}\right)-\sum_{i=1}^{n}\left(\frac{G^{\prime}\left(x_{i}, \phi\right)}{\bar{G}\left(x_{i}, \phi\right)}\right)+2(\theta-1) \sum_{i=1}^{n}\left\{\frac{\bar{G}\left(x_{i}, \phi\right) G^{\prime}\left(x_{i}, \phi\right)}{a_{i}}\right\} \\
& +2 \lambda \theta(\alpha-1) \sum_{i=1}^{n}\left\{\frac{\bar{G}\left(x_{i}, \phi\right) G^{\prime}\left(x_{i}, \phi\right) a_{i}^{\theta-1}}{1+\lambda a_{i}^{\theta}}\right\} \\
& -2 \lambda \theta \alpha \sum_{i=1}^{n}\left\{\bar{G}\left(x_{i}, \phi\right) G^{\prime}\left(x_{i}, \phi\right) a_{i}^{\theta-1}\left(1+\lambda a_{i}^{\theta}\right)^{\alpha-1}\right\} .
\end{aligned}
$$

where, $g^{\prime}\left(x_{i}, \phi\right)=\partial g\left(x_{i}, \phi\right) / \partial \phi$ and $G^{\prime}\left(x_{i}, \phi\right)=\partial G\left(x_{i}, \phi\right) / \partial \phi$.

Equating (31), (32), (33) and (34) to zero and solving for $\alpha, \lambda, \theta$ and $\phi$ simultaneously yields the MLEs, say $\hat{\Phi}=(\hat{\alpha}, \hat{\lambda}, \hat{\theta}, \hat{\phi})$ of $\Phi=(\alpha, \lambda, \theta, \phi)^{T}$. The components of the observed information matrix are derived in Appendix A.

\section{Applications}

In this section, we provide two applications to real data by using the NHTLLx distribution, which serve to illustrate the importance of the NHTL-G family.

The first real data set consists of 63 observations of the strengths of $1.5 \mathrm{~cm}$ glass fibres which obtained by workers at the UK National Physical Laboratory. The data are: 0.55, $0.74,0.77,0.81,0.84,0.93,1.04,1.11,1.13,1.24,1.25,1.27,1.28,1.29,1.30,1.36,1.39$, $1.42,1.48,1.48,1.49,1.49,1.50,1.50,1.51,1.52,1.53,1.54,1.55,1.55,1.58,1.59,1.60$, $1.61,1.61,1.61,1.61,1.62,1.62,1.63,1.64,1.66,1.66,1.66,1.67,1.68,1.68,1.69,1.70$, $1.70,1.73,1.76,1.76,1.77,1.78,1.81,1.82,1.84,1.84,1.89,2.00,2.01,2.24$.

The second data set represents 13 observations of cotton average yield in different years in Lodhran district in Pakistan, see Beig (2017). The data are: 26.90, 24.16, 21.52, $16.75,19.37,17.46,23.97,21.92,21.38,23.73,12.50,20.69,20.00$. These two datasets are recently studied by (Reyad et al. 2018b).

Initially, we fit the NHTLLx model to these data sets and compare it with some competitive models such as; beta Lomax (BLx) (Lemonte and Cordeiro 2013), Kumaraswamy Lomax (KwLx) (Shams 2013), exponentiated Lomax (ELx) (AbdulMoniem and Abdel-Hameed 2012), Odd-Lindley-Lomax (OLLx) (Gomes-Silva et al. 2017), Topp-Leone Lomax (TLLx) (Al-Shomrani et al. 2016), Lomax (Lx) and Nadarajah Haghighi (NH) (Nadarajah and Haghighi 2011) distributions.

In order to compare these models and to verify the quality of the fits, we consider some of the well-known goodness-of-fit statistics like, Cramér-von Mises $\left(W^{*}\right)$, Anderson Darling $\left(A^{*}\right)$, Kolmogorov-Smirnov $(K S)$, -Loglikelihood $(-L)$, Akaike Information 
Criterion $(A I C)$, Consistent Akaike Information Criterion $(C A I C)$, Bayesian information criterion (BIC) and Hannan-Quinn criterion (HQC). The model with a minimum value of Goodness-of-Fit statistics is the best model to fit the data. The required numerical evaluations are implemented using the BFGS quasi-Newton method in R software.

The results of the MLEs and their standard errors of the fitted models for the two data sets are displayed in Tables 1 and 3 respectively. In addition, the values of $W^{*}, A^{*}$, $K S,-L, A I C, C A I C, B I C$ and HOIQ statistics of all fitted models for the two data sets are displayed in Tables 2 and 4 respectively.

Table 1: The MLEs and their standard errors (in parentheses) for the first data set

\begin{tabular}{|c|c|c|c|c|c|}
\hline \multirow{2}{*}{ Models } & \multicolumn{5}{|c|}{ Estimates } \\
\hline & $a$ & $b$ & $\alpha$ & $\lambda$ & $\theta$ \\
\hline NHTLLx & $\begin{array}{c}0.9152 \\
(1.1542)\end{array}$ & $\begin{array}{c}0.2090 \\
(0.2762)\end{array}$ & $\begin{array}{c}25.0302 \\
(46.8917) \\
\end{array}$ & $\begin{array}{c}5.9320 \\
(36.5898) \\
\end{array}$ & $\begin{array}{c}6.1208 \\
(8.1393) \\
\end{array}$ \\
\hline BLx & $\begin{array}{l}17.8478 \\
(3.4499) \\
\end{array}$ & $\begin{array}{c}40.1913 \\
(60.2638) \\
\end{array}$ & $\begin{array}{c}77.5816 \\
(222.1545)\end{array}$ & $\begin{array}{c}19.3027 \\
(41.0907) \\
\end{array}$ & --- \\
\hline KwLx & $\begin{array}{c}27.1531 \\
(67.0525) \\
\end{array}$ & $\begin{array}{c}46.1506 \\
(125.0250) \\
\end{array}$ & $\begin{array}{c}9.0710 \\
(2.3638) \\
\end{array}$ & $\begin{array}{c}91.7567 \\
(89.5981) \\
\end{array}$ & --- \\
\hline ELx & $\begin{array}{c}43.2618 \\
(53.4628)\end{array}$ & $\begin{array}{c}116.1829 \\
(139.3477)\end{array}$ & $\begin{array}{c}32.7701 \\
(10.3498) \\
\end{array}$ & --- & --- \\
\hline OLLx & $\begin{array}{c}1.8079 \\
(0.1482) \\
\end{array}$ & $\begin{array}{c}79.9901 \\
(22.3521) \\
\end{array}$ & $\begin{array}{l}29.5177 \\
(8.8291) \\
\end{array}$ & --- & --- \\
\hline TLLx & $\begin{array}{c}44.4017 \\
(54.9352)\end{array}$ & $\begin{array}{c}59.6955 \\
(72.1453) \\
\end{array}$ & $\begin{array}{c}32.8566 \\
(10.1778) \\
\end{array}$ & --- & --- \\
\hline Lx & $\begin{array}{c}211.2298 \\
(322.7742) \\
\end{array}$ & $\begin{array}{c}140.5679 \\
(214.5268) \\
\end{array}$ & -- & --- & --- \\
\hline $\mathrm{NH}$ & $\begin{array}{c}139.801 \\
(66.6059) \\
\end{array}$ & $\begin{array}{c}0.0037 \\
(0.0017) \\
\end{array}$ & --- & --- & --- \\
\hline
\end{tabular}

Table 2: Goodness of fit statistics for all fitted models for the first data set.

\begin{tabular}{cccccccccc}
\hline Models & \multirow{2}{*}{$A^{*}$} & $W^{*}$ & $-L$ & AIC & CAIC & BIC & HQC & K-S & $\begin{array}{c}\text { P- } \\
\text { value }\end{array}$ \\
\hline NHTLLx & 1.2323 & 0.2207 & 15.5482 & 41.097 & 42.149 & 51.8122 & 45.311 & 0.1548 & 0.0974 \\
\hline BLx & 3.1526 & 0.57484 & 24.1456 & 56.291 & 56.981 & 64.864 & 59.663 & 0.2165 & 0.0054 \\
\hline KwLx & 1.7966 & 0.3281 & 17.2688 & 42.538 & 43.227 & 51.11 & 45.909 & 0.1731 & 0.0457 \\
\hline ELx & 4.3439 & 0.7969 & 31.7373 & 69.475 & 69.881 & 75.904 & 72.003 & 0.2285 & 0.0027 \\
\hline OLLx & 2.4574 & 0.4481 & 69.9755 & 145.951 & 146.358 & 152.38 & 148.48 & 0.3724 & 0.0000 \\
\hline TLLx & 4.3452 & 0.7972 & 31.7284 & 69.457 & 69.864 & 75.886 & 71.986 & 0.2300 & 0.0025 \\
\hline Lx & 3.1354 & 0.5717 & 89.0439 & 182.088 & 182.288 & 186.374 & 183.774 & 0.4179 & 0.000 \\
\hline NH & 2.3152 & 0.4223 & 68.7617 & 141.523 & 141.723 & 145.810 & 143.209 & 0.4519 & 0.000 \\
\hline
\end{tabular}



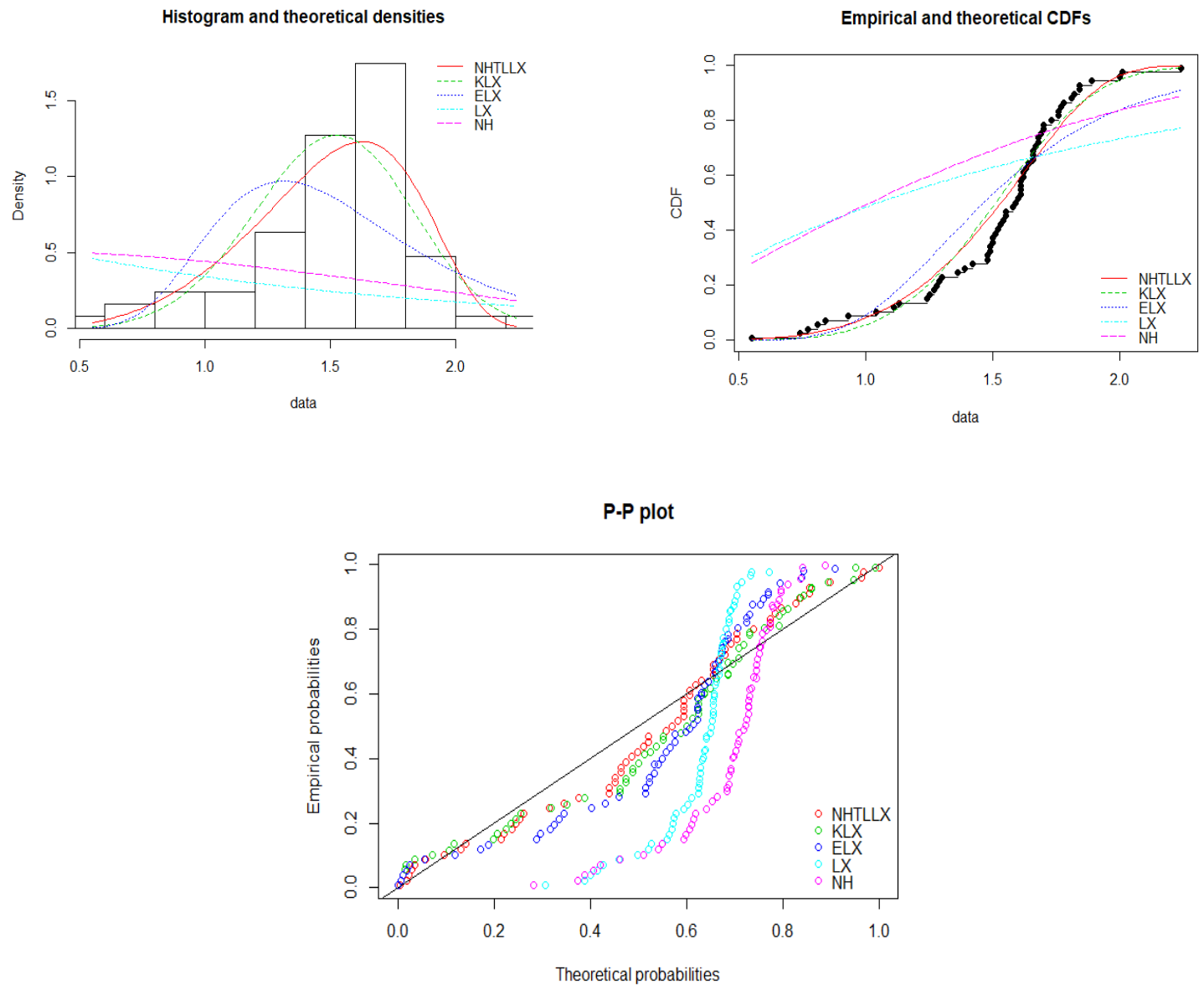

Figure. 4: Histogram and estimated densities (left upper panel); Theoretical and estimated CDFs (right upper panel); P-P plots (lower panel) for the first data set.

Table 3: The MLEs and their standard errors (in parentheses) for the second data set

\begin{tabular}{|c|c|c|c|c|c|}
\hline \multirow{2}{*}{ Models } & \multicolumn{5}{|c|}{ Estimates } \\
\hline & $a$ & $b$ & $\alpha$ & $\lambda$ & $\theta$ \\
\hline NHTLLx & $\begin{array}{c}3.6485 \\
(4.9110)\end{array}$ & $\begin{array}{c}0.0146 \\
(0.0021)\end{array}$ & $\begin{array}{c}3.6599 \\
(6.4367)\end{array}$ & $\begin{array}{c}3.7324 \\
(15.4892)\end{array}$ & $\begin{array}{c}20.9750 \\
(32.7064)\end{array}$ \\
\hline BLx & $\begin{array}{c}36.2399 \\
(28.3572)\end{array}$ & $\begin{array}{c}30.4202 \\
(93.6734)\end{array}$ & $\begin{array}{c}58.1401 \\
(147.5679)\end{array}$ & $\begin{array}{c}2.5363 \\
(7.2874)\end{array}$ & --- \\
\hline KwLx & $\begin{array}{c}2.8402 \\
(1.3255) \\
\end{array}$ & $\begin{array}{c}20.2466 \\
(13.6450) \\
\end{array}$ & $\begin{array}{l}18.5246 \\
(9.4360) \\
\end{array}$ & $\begin{array}{c}9.4511 \\
(6.1925) \\
\end{array}$ & --- \\
\hline ELx & $\begin{array}{c}232.6174 \\
(511.1264)\end{array}$ & $\begin{array}{c}59.5700 \\
(121.6153)\end{array}$ & $\begin{array}{c}115.4825 \\
(109.1986)\end{array}$ & --- & --- \\
\hline OLLx & $\begin{array}{l}12.6601 \\
(4.1220)\end{array}$ & $\begin{array}{l}11.2461 \\
(9.2156)\end{array}$ & $\begin{array}{c}0.0785 \\
(2.3131)\end{array}$ & --- & --- \\
\hline TLLx & $\begin{array}{c}188.5994 \\
(379.5414)\end{array}$ & $\begin{array}{c}24.5373 \\
(45.1973)\end{array}$ & $\begin{array}{c}119.1881 \\
(114.7701)\end{array}$ & --- & --- \\
\hline Lx & $\begin{array}{c}235.7117 \\
(229.6951)\end{array}$ & $\begin{array}{c}11.3362 \\
(10.8514)\end{array}$ & --- & --- & --- \\
\hline $\mathrm{NH}$ & $\begin{array}{c}9.7418 \\
(6.7881)\end{array}$ & $\begin{array}{c}0.0040 \\
(0.0028)\end{array}$ & --- & --- & --- \\
\hline
\end{tabular}


Table 4: Goodness of fit statistics for all fitted models for the second data set.

\begin{tabular}{cccccccccc}
\hline Model & $A^{*}$ & $W^{*}$ & $-L$ & AIC & CAIC & BIC & HQC & K-S & $\begin{array}{c}\text { P- } \\
\text { value }\end{array}$ \\
\hline NHTLLx & 0.2175 & 0.0311 & 34.8134 & 79.627 & 88.198 & 82.452 & 79.046 & 0.1012 & 0.9973 \\
\hline BLx & 0.3986 & 0.0648 & 40.0340 & 88.068 & 93.068 & 90.328 & 87.604 & 0.1745 & 0.7248 \\
\hline KwLx & 0.3613 & 0.0591 & 40.9954 & 89.991 & 94.991 & 92.251 & 89.526 & 0.1979 & 0.5758 \\
\hline ELx & 0.6169 & 0.0985 & 41.2033 & 88.407 & 91.073 & 90.101 & 88.058 & 0.1993 & 0.5672 \\
\hline OLLx & 0.4397 & 0.0710 & 62.9155 & 131.831 & 134.498 & 133.526 & 131.483 & 0.5111 & 0.0006 \\
\hline TLLx & 0.6215 & 0.0993 & 41.2299 & 88.46 & 91.126 & 90.155 & 88.111 & 0.1996 & 0.5652 \\
\hline Lx & 0.3687 & 0.0602 & 57.5090 & 119.018 & 120.218 & 120.148 & 118.786 & 0.4693 & 0.0023 \\
\hline NH & 0.3090 & 0.0448 & 48.6385 & 101.277 & 102.477 & 102.407 & 101.045 & 0.5094 & 0.0012 \\
\hline
\end{tabular}
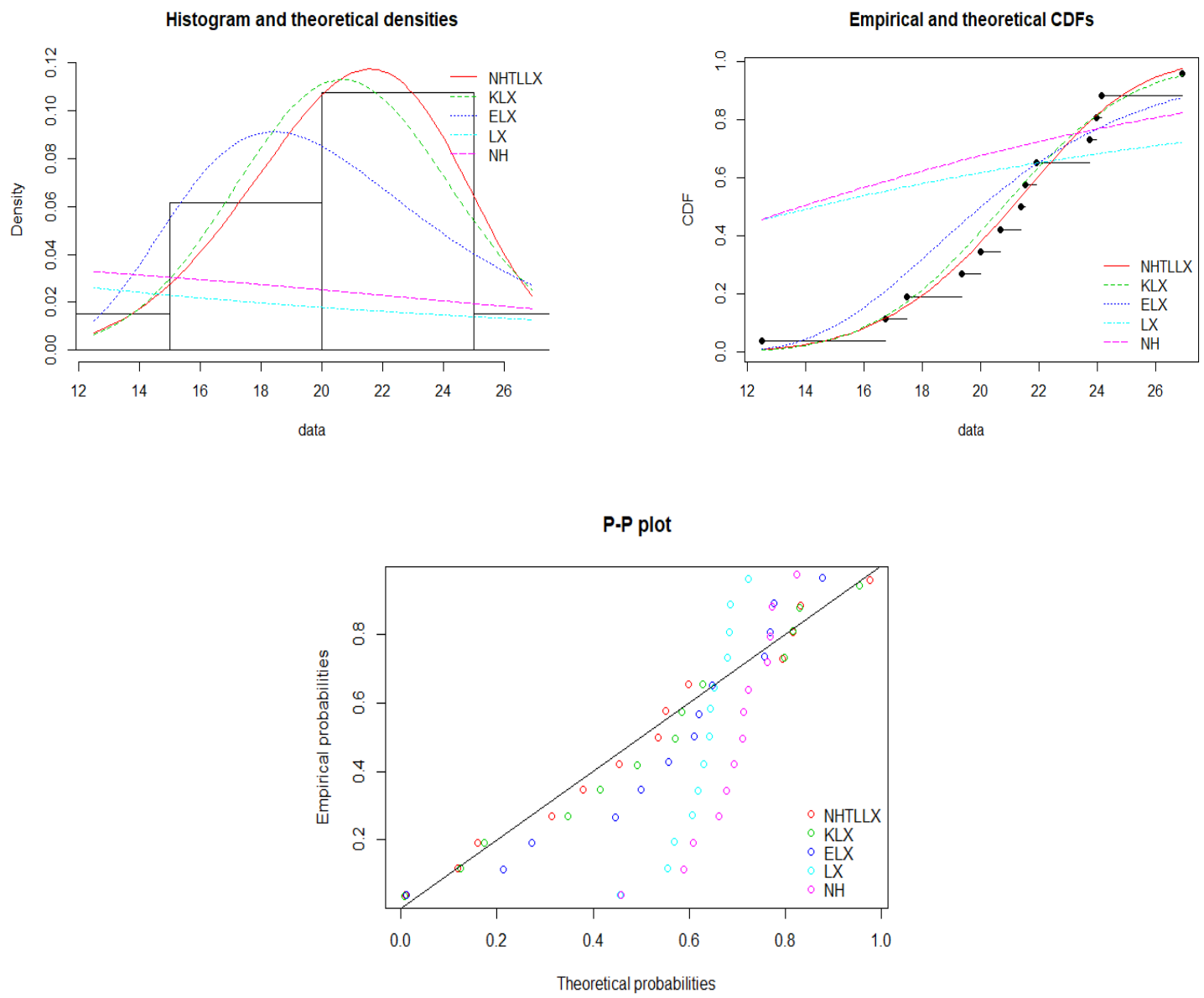

Figure. 5: Histogram and estimated densities (left upper panel); Theoretical and estimated CDFs (right upper panel); P-P plots (lower panel) for the second data set.

The values in Tables 2, 4 show that the NHTLLx distribution gives the smallest values for the Goodness-of-Fit statistics and the greatest of p-value of KS-test. This means that NHTLLx distribution provides better fit than the rest of the distributions. Additionally, Figures 4, 5 display the plots of fitted and empirical densities of competitive distributions for datasets I and II, respectively. These figures confirm that the NHTLLx distribution is more appropriate to represent the two datasets than the rest distributions. 


\section{Conclusions}

We propose and study a new generator of continuous distributions, also obtained. We introduce two real applications to show the importance of the new family called the Nadarajah Haghighi Topp Leone-G family based on the Nadarajah Haghighi distribution, the T-X and TL-G classes. We provide three sub-models corresponding to the new family and derive an useful expansion of its density and distribution functions in terms of the exponential-G class. Moreover, we introduc an extensive treatment of the mathematical properties of the NHTL$\mathrm{G}$ family such as quantile function, raw and incomplete moments, order statistics, stress strenght model, generating functions, entropies and probability weighted moments. The model parameters of the new family are estimated via maximum likelihood estimation and the observed information matrix is obtained. Two real applications are provided to show the effectivness of the new family. Numerical calculations showed that some sub-models of the NHTL-G family can give better fit than similar models generated by other families.

\section{References}

1. Aldahlan, M. A., Afify, A, Z. and Ahmed, A. N. (2019). The odd inverse Pareto-G class: properties and applications. Journal of Nonlinear Sciences and Applications; 12: 278-290.

2. Alizadeh, M., Tahmasebi, S. and Haghbin, H. (2018). The exponentiated odd logLogistic family of distributions: properties and applications. Journal of Statistical Modeling:Theory and Applications; 1(2): 1-24.

3. Alzaatreh, A, Lee, C. and Famoye, F. (2013). A new method for generating families of continuous distributions. Metron; 71(1):63-79.

4. Al-Shomrani, A., Arif, O., Shawky, K., Hanif, S. and Shahbaz, M. Q. (2016). ToppLeone family of distributions: some properties and application, Pak.j.stat.oper.res.; 12:443-451.

5. Abdul-Moniem, I., and H. Abdel-Hameed. (2012). ON exponentiated Lomax distribution. International Journal of Mathematical Archive EISSN 2229-5046 3 (5).

6. Beig, M. A. (2017). Crop Reporting Service, Multan Road, Lahore, Pakistan. Director crop Reporting Service www.crs.agripunjab.gov.pk.

7. Cordeiro, G. M., Afify, A. Z., Ortega, E. M. M., Suzuki, A. K. and Mead, M. E. (2019). The odd Lomax generator of distributions: properties, estimation and applications. Journal of Computational and Applied Mathematics; 347:222-237.

8. Lemonte, A., Cordeiro, G. (2013). An extended lomax distribution, Statistics, 47: 800-816.

9. Gomes-Silva, F. S., A. Percontini, E. de Brito, M. W. Ramos, R. Venâncio, and Cordeiro, G. M. (2017). The odd Lindley-G family of distributions. Austrian Journal of Statistics 46 (1):65-87.

10. Korkmaz, M. C., Alizadeh, M., Yousof, H.M. and Butt, N. S. (2018b). The generalized odd Weibull generated family of distributions: statistical properties and applications. Pak.j.stat.oper.res. vol.XIV. No. 3:541-556. 
11. Korkmaz, M. C., Yousof, H. M., Hamedani, G. G. and Ali, M. M. (2018a). The Marshall-Olkin generalized G Poison family of distributions. Pakistan Journal of Statistics; 34(3):251-267.

12. Mazed, N., Kumar, D., Dey, S. and Cordeiro, G. M. (2019). The Marshall-Plkin alpha power family of distributions with applications. Journal of Computational and Applied Mathematics; 351: 41-53.

13. Nadarajah, S., and F. Haghighi. (2011). An extension of the exponential distribution. Statistics 45 (6):543-558.

14. Reyad, H., Jamal, F., Othman, S. and Hamedani, G. G. (2018a). The transmuted Gompertz-G family of distributions: properties and applications. Tbilist Mathematical Journal; 11(3): 47-67.

15. Reyad, H., Alizadeh, M., Jamal, F. and Othman, S. (2018b). The Topp Leone odd Lindley-G family of distributions: properties and applications. Journal of Statistics and Management Systems; 21(7): 1273-1297.

16. Reyad, H., Alizadeh, M., Jamal, F., Othman, S. and Hamedani, G. G (2019b). The exponentiated generalized Topp Leone-G family of distributions: theory and applications. Pak.j.stat.res. XV (1); 1-24.

17. Reyad, H., Othman, S. and Ul Haq, M. A. (2019a). The transmuted generalized odd generalized exponential-G family of distributions: theory and applications. Journal of Data science; 17(2):279-300.

18. Shams, T. (2013). The Kumaraswamy-generalized Lomax distribution. Middle-East Journal of Scientific Research; 17 (5):641-646.

19. Yousof, H. M., Afify, A. Z., Alizadeh, M., Hamedani, G. G., Jahanshahi, S. M. A. and Ghosh, I. (2018). The generalized transmuted Poisson-G family of distributions. Pak.j.stat.res. XIV (4); 759-779.

20. Yousof, H. M., Rasekhi, M., Altun, E., \& Alizadeh, M. (2019). The extended odd Fréchet family of distributions: properties, applications and regression modeling. Int J Math Comput, 30, 1-16.

\section{Appendix A}

The observed information matrix has the following components:

$$
\begin{aligned}
& \frac{\partial^{2} \ell}{\partial \alpha^{2}}=\frac{-n}{\alpha^{2}}-\sum_{i=1}^{n}\left\{\left(1+\lambda a_{i}^{\theta}\right)^{\alpha}\left[\log \left(1+\lambda a_{i}^{\theta}\right)\right]^{2}\right\}, \\
& \frac{\partial^{2} \ell}{\partial \lambda \partial \alpha}=\sum_{i=1}^{n}\left\{\frac{a_{i}^{\theta}}{1+\lambda a_{i}^{\theta}}\right\}-\sum_{i=1}^{n}\left\{a_{i}^{\theta}\left(1+\lambda a_{i}^{\theta}\right)^{\alpha-1}\left[1+\log \left(1+\lambda a_{i}^{\theta}\right)^{\alpha}\right]\right\}, \\
& \frac{\partial^{2} \ell}{\partial \theta \partial \alpha}=\lambda \sum_{i=1}^{n}\left\{\frac{a_{i}^{\theta} \log \left(a_{i}\right)}{1+\lambda a_{i}^{\theta}}\right\}-\sum_{i=1}^{n}\left\{a_{i}^{\theta}\left(1+\lambda a_{i}^{\theta}\right)^{\alpha-1} \log \left(a_{i}\right)\left[1+\log \left(1+\lambda a_{i}^{\theta}\right)^{\alpha}\right]\right\}, \\
& \frac{\partial^{2} \ell}{\partial \phi \partial \alpha}=2 \lambda \theta \sum_{i=1}^{n}\left\{\frac{\bar{G}\left(x_{i}, \phi\right) G^{\prime}\left(x_{i}, \phi\right) a_{i}^{\theta-1}}{1+\lambda a_{i}^{\theta}}\right\}
\end{aligned}
$$




$$
\begin{aligned}
& -2 \lambda \theta \sum_{i=1}^{n}\left\{\bar{G}\left(x_{i}, \phi\right) G^{\prime}\left(x_{i}, \phi\right) a_{i}^{\theta-1}\left(1+\lambda a_{i}^{\theta}\right)^{\alpha-1}\left[1+\log \left(1+\lambda a_{i}^{\theta}\right)^{\alpha}\right]\right\}, \\
& \frac{\partial^{2} \ell}{\partial \lambda^{2}}=\frac{-n}{\lambda^{2}}-(\alpha-1) \sum_{i=1}^{n}\left\{\frac{a_{i}^{2 \theta}}{\left(1+\lambda a_{i}^{\theta}\right)^{2}}\right\}-\alpha(\alpha-1) \sum_{i=1}^{n}\left\{a_{i}^{2 \theta}\left(1+\lambda a_{i}^{\theta}\right)^{\alpha-2}\right\} \\
& \frac{\partial^{2} \ell}{\partial \theta \partial \lambda}=(\alpha-1) \sum_{i=1}^{n}\left\{\frac{a_{i}^{\theta} \log \left(a_{i}\right)}{\left(1+\lambda a_{i}^{\theta}\right)^{2}}\right\}+(\alpha-1) \sum_{i=1}^{n}\left\{a_{i}^{\theta}\left\{1+(\lambda+\alpha-1) a_{i}^{\theta}\right\} \log \left(a_{i}\right)\left(1+\lambda a_{i}^{\theta}\right)^{\alpha-2}\right\}, \\
& \frac{\partial^{2} \ell}{\partial \phi \partial \lambda}=2 \theta(\alpha-1) \sum_{i=1}^{n}\left\{\frac{\bar{G}\left(x_{i}, \phi\right) G^{\prime}\left(x_{i}, \phi\right) a_{i}^{\theta-1}}{\left(1+\lambda a_{i}^{\theta}\right)^{2}}\right\} \\
& -2 \alpha \theta \sum_{i=1}^{n}\left\{\bar{G}\left(x_{i}, \phi\right) G^{\prime}\left(x_{i}, \phi\right) a_{i}^{\theta-1}\left(1+\lambda a_{i}^{\theta}\right)^{\alpha-2}\left(1+\alpha \lambda a_{i}^{\theta}\right)\right\} \\
& \frac{\partial^{2} \ell}{\partial \theta^{2}}=\frac{-n}{\theta^{2}}+\lambda(\alpha-1) \sum_{i=1}^{n}\left\{\frac{a_{i}^{\theta}\left(\log \left(a_{i}\right)\right)^{2}}{\left(1+\lambda a_{i}^{\theta}\right)^{2}}\right\}-\lambda \alpha \sum_{i=1}^{n}\left\{a_{i}^{\theta}\left(\log \left(a_{i}\right)\right)^{2}\left(1+\alpha \lambda a_{i}^{\theta}\right)\left(1+\lambda a_{i}^{\theta}\right)^{\alpha-2}\right\}, \\
& \frac{\partial^{2} \ell}{\partial \phi \partial \theta}=2 \sum_{i=1}^{n}\left\{\frac{\bar{G}\left(x_{i}, \phi\right) G^{\prime}\left(x_{i}, \phi\right)}{a_{i}}\right\}+2 \lambda(\alpha-1) \sum_{i=1}^{n}\left\{\frac{\bar{G}\left(x_{i}, \phi\right) G^{\prime}\left(x_{i}, \phi\right) a_{i}^{\theta-1}\left[1+\lambda a_{i}^{\theta}+\theta \log \left(a_{i}\right)\right]}{\left(1+\lambda a_{i}^{\theta}\right)^{2}}\right\} \\
& -2 \alpha \lambda \sum_{i=1}^{n}\left\{\bar{G}\left(x_{i}, \phi\right) G^{\prime}\left(x_{i}, \phi\right) a_{i}^{\theta-1}\left(1+\lambda a_{i}^{\theta}\right)^{\alpha-2}\left[1+\theta+\lambda(1+\theta \alpha) a_{i}^{\theta}\right]\right\}, \\
& \frac{\partial^{2} \ell}{\partial \phi^{2}}=\sum_{i=1}^{n}\left(\frac{g\left(x_{i}, \phi\right) g^{\prime \prime}\left(x_{i}, \phi\right)-g^{\prime}\left(x_{i}, \phi\right)^{2}}{g\left(x_{i}, \phi\right)^{2}}\right)-\sum_{i=1}^{n}\left(\frac{\bar{G}\left(x_{i}, \phi\right) G^{\prime \prime}\left(x_{i}, \phi\right)+G^{\prime}\left(x_{i}, \phi\right)^{2}}{\bar{G}\left(x_{i}, \phi\right)^{2}}\right) \\
& -2(\theta-1) \sum_{i=1}^{n}\left\{\frac{a_{i} b_{i}-2 c_{i}}{a_{i}^{2}}\right\}+2 \lambda \theta(\alpha-1) \sum_{i=1}^{n}\left\{\frac{a_{i}^{\theta-2} z_{i}}{\left(1+\lambda a_{i}^{\theta}\right)^{2}}\right\} \\
& +2 \lambda \theta \alpha \sum_{i=1}^{n}\left\{a_{i}^{\theta-2}\left(\rho_{i}-v_{i}\right)\left(1+\lambda a_{i}^{\theta}\right)^{\alpha-2}\right\}
\end{aligned}
$$

where,

$$
\begin{aligned}
& b_{i}=\bar{G}\left(x_{i}, \phi\right) G^{\prime \prime}\left(x_{i}, \phi\right)-G^{\prime}\left(x_{i}, \phi\right)^{2}, \\
& c_{i}=\bar{G}\left(x_{i}, \phi\right)^{2} G^{\prime}\left(x_{i}, \phi\right)^{2}, \\
& z_{i}=\left(1+\lambda a_{i}^{\theta}\right)\left[2(\theta-1) c_{i}+a_{i} b_{i}\right]-2 \lambda \theta c_{i} a_{i}^{\theta}, \\
& \rho_{i}=2 c_{i}\left\{1-\theta+\lambda(1-\theta \alpha) a_{i}^{\theta}\right\}, \\
& v_{i}=a_{i} b_{i}\left(1+\lambda a_{i}^{\theta}\right), \\
& g^{\prime \prime}\left(x_{i}, \phi\right)=\partial^{2} g\left(x_{i}, \phi\right) / \partial \phi^{2} \\
& \text { and }
\end{aligned}
$$$$
G^{\prime \prime}\left(x_{i}, \phi\right)=\partial^{2} G\left(x_{i}, \phi\right) / \partial \phi^{2} \text {. }
$$ 